\title{
A randomised controlled trial of two methods of delivering nasal continuous positive airway pressure after extubation to infants weighing less than 1000 g: binasal (Hudson) versus single nasal prongs
}

P Davis, M Davies, B Faber

\begin{abstract}
Objectives-Primary: to determine whether nasal continuous positive airway pressure (CPAP) delivered through binasal prongs results in a greater proportion of extremely low birthweight infants being successfully extubated, after a period of intermittent positive pressure ventilation, than nasal CPAP delivered by a single nasal prong. Secondary: to evaluate the effect of mode of delivery of nasal CPAP after extubation on the need for endotracheal reintubation, weight gain, rates of feeding intolerance, sepsis, suspected sepsis, cranial ultrasound abnormalities, retinopathy of prematurity, chronic lung disease, and the duration of assisted ventilation and care in the tertiary neonatal unit.

Design and setting-Randomised, controlled, clinical trial conducted at the neonatal intensive care unit of the Royal Women's Hospital, Melbourne, Australia. Patients-Infants of birth weight less than $1000 \mathrm{~g}$, ventilated, requiring $<50 \%$ oxygen and ventilator rate less than or equal to $20 /$ minute, and considered by the clinical management team to be ready for extubation.
\end{abstract}

Intervention-Infants were randomly allocated to receive nasal CPAP delivered through binasal (Hudson) prongs or a single nasal prong.

Primary outcome measure-Failure of extubation as defined by the following criteria: $(a)$ apnoea (more than one episodel hour over a six hour period or one episode requiring bag and mask ventilation); $(b)$ absolute increase in oxygen requirement greater than $15 \%$ above that required before extubation; (c) respiratory acidosis (pH $<7.25$ with $\left.\mathrm{PCO}_{2}>6.67 \mathrm{kPa}\right)$.

Results-Ten of the 41 (24\%) infants randomised to binasal prongs reached predetermined failure criteria compared with 26 of the $46(57 \%)$ infants randomised to a single nasal prong $(p=0.005)$. Four of
$17(24 \%)$ infants of birth weight less than $800 \mathrm{~g}$ extubated to binasal prongs reached failure criteria compared with 14 of 16 (88\%) extubated to a single nasal prong $(p<0.001)$. There were no significant differences in any of the secondary outcomes.

Conclusions-For extremely low birthweight infants ventilated using an endotracheal tube, nasal CPAP delivered through binasal (Hudson) prongs is more effective in preventing failure of extubation than that delivered through a single nasal prong.

(Arch Dis Child Fetal Neonatal Ed 2001;85:F82-F85)

Keywords: ventilation; continuous positive airway pressure; extremely low birth weight infant; extubation

Current management of extremely low birth weight (ELBW) infants - that is, those of birth weight less than $1000 \mathrm{~g}$-often includes insertion of an endotracheal tube, positive pressure ventilation, and exogenous surfactant administration. Although these treatments may be life saving, prolonged ventilation is associated with the development of chronic lung disease, pulmonary and systemic infections, and damage to the upper airways. Early removal of the endotracheal tube is thought desirable. A systematic review has shown that the use of nasal continuous positive airway pressure (CPAP) is effective in preventing failure of extubation when compared with headbox oxygen. ${ }^{1}$ Meta-analysis of seven randomised trials showed that rates of extubation failure for infants randomised to nasal CPAP ranged from $22 \%$ to $52 \%$, with a pooled estimate of $34 \% .{ }^{1}$ Several methods of delivering nasal CPAP were used in the studies in this systematic review but there have been no published randomised trials comparing extubation failure rates of one method of CPAP delivery with another. We conducted a prospective randomised trial to determine whether CPAP delivered by binasal (Hudson) prongs resulted in a greater proportion of infants being successfully extubated, 


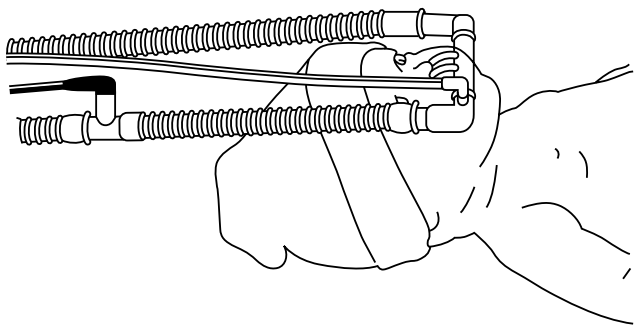

Figure 1 Hudsons prongs extending about $1 \mathrm{~cm}$ inside the nose.

without unwanted side effects, compared with management with a single short nasal prong.

\section{Methods}

All babies (inborn and outborn) admitted to the neonatal intensive care unit at the Royal Women's Hospital, Melbourne, Australia were considered for participation in this trial. Eligibility criteria were (a) birth weight less than $1000 \mathrm{~g},(b)$ endotracheal intubation for any duration, (c) stable or improving respiratory status with a ventilator rate less than or equal to 20/minute and an inspired oxygen concentration of less than 50\%, and (d) considered by the clinical management team to be ready for extubation. The mode of CPAP delivery was randomly allocated (using a random number table and sequentially numbered, sealed, opaque envelopes) after informed parental consent had been obtained. Infants were stratified by sex and birth weight (<800 g and 800-999 g). Variable block sizes were used within each stratum.

All infants received a loading dose of theophylline and were extubated from low rate ventilation rather than endotracheal CPAP or high frequency oscillation. The sealed envelope was opened immediately before extubation and infants were allocated to receive nasal CPAP through either a single nasal prong or binasal Hudson prongs. Single prong CPAP was administered through a size 2.5 or 3.0 Portex tube (Portex Ltd, Hyth, Kent, UK) inserted $2.5 \mathrm{~cm}$ into one nostril and connected to a ventilator. Binasal CPAP was administered through a Hudson infant nasal CPAP cannula (Hudson Respiratory Care Inc, Temecula, California, USA) (fig 1). Ventilator flow rates were set at 6 litres/minute, and the CPAP level was set at $7 \mathrm{~cm}$ water initially in both groups. CPAP was decreased according to the infant's status to a level of $5 \mathrm{~cm}$ water before discontinuation. In both groups concentrations of inspired oxygen were adjusted to maintain oxygen saturation levels at 90-95\%. Analysis of capillary or arterial blood gases was performed six hours after extubation and then at least daily for the week after extubation.

PRIMARY OUTCOME

Successful extubation was determined over the seven days after removal of the endotracheal tube. An infant was deemed to have failed if any of the following were experienced:

- apnoea: minor (more than six episodes requiring physical stimulation over a six hour period) or major (one episode requiring bag and mask ventilation);
- an absolute increase in oxygen concentration of greater than $15 \%$ above that required immediately before extubation;

- respiratory acidosis- $\mathrm{pH}<7.25$ with $\mathrm{PCO}_{2}>6.67 \mathrm{kPa}$.

SECONDARY OUTCOMES

Endotracheal reintubation after occurrence of failure criteria was at the attending clinician's discretion and this event was measured as a secondary outcome. Weight gain over the week after extubation and over the period from extubation to discharge from hospital were measured. Feeding intolerance was defined as the presence of a fourth hourly gastric aspirate greater than $25 \%$ of the feed volume or containing bile during the week after extubation. Sepsis was diagnosed on the basis of a positive culture of blood, cerebrospinal fluid, or bladder tap urine. Suspected sepsis was defined as a culture negative episode, treated with at least five days of antibiotics in conjunction with an immature to total neutrophil ratio of greater than 0.15. Cranial ultrasound examinations were performed within the first three days of life, on days 7 and 28, and then monthly until discharge; cerebroventricular haemorrhage was classified as described by Papile et al. ${ }^{2}$ Infants were examined every two weeks by an experienced ophthalmologist, and retinopathy of prematurity classified according to the International Classification of Retinopathy of Prematurity. ${ }^{3}$ Chronic lung disease was determined by supplemental oxygen requirement at 28 days of age or 36 weeks corrected age. Other secondary outcomes included the duration of assisted ventilation and care in the tertiary neonatal unit.

A sample size of 130 was chosen to provide a power of $80 \%$ to detect a reduction in failure rate from $50 \%$ to $25 \%$ with a two tailed $\mathrm{p}$ of less than 0.05 . Interim analyses by an external data monitoring committee were planned after enrolment of 43 and 87 patients. According to the O'Brien Fleming procedure ${ }^{4}$, the $\mathrm{p}$ values to stop the trial were $<0.0006$ after one third of the patients entered and $<0.015$ after two thirds. $\chi^{2}$ analysis was used for comparison of failure rates and other categorical patient variables. A two tailed Student's $t$ test and MannWhitney $U$ test were used for continuous data as appropriate.

The research and ethics committees of the Royal Women's Hospital, Melbourne, Victoria, Australia approved the study.

\section{Results}

From October 1997 through July 1999, 164 infants of birth weight less than $1000 \mathrm{~g}$ were admitted to the neonatal intensive care unit. Of these, 23 were never intubated, 27 died before extubation, and four were transferred to other hospitals because of bed shortages. Reasons for non-enrolment of the remainder included consent refusal by the parents (6) or neonatologist (2) and accidental extubation before consent had been sought (15). The remaining 87 infants were randomised to receive either binasal (41) or single (46) nasal prongs. 
Table 1 Patient characteristics

\begin{tabular}{lll}
\hline & Binasal $(n=41)$ & Single $(n=46)$ \\
\hline Birth weight $(\mathrm{g})^{\star}$ & $790(140)$ & $816(125)$ \\
Gestational age (weeks) ${ }^{\star}$ & $26(1.9)$ & $26(1.9)$ \\
Age at extubation (days) $\dagger$ & $3(1-9)$ & $3(1-6)$ \\
Inborn/outborn & $37 / 4$ & $37 / 9$ \\
Male/female & $21 / 20$ & $24 / 22$ \\
Single/multiple & $32 / 9$ & $31 / 15$ \\
Any maternal steroids & $40(98 \%)$ & $44(96 \%)$ \\
Surfactant & $39(95 \%)$ & $43(93 \%)$ \\
Inspired oxygen concentration $(\%)$ at extubation $\dagger$ & $21(21-21)$ & $21(21-25)$ \\
Rate at extubation (breaths/minute) $\dagger$ & $20(15-20)$ & $20(15-20)$ \\
Mean airway pressure at extubation $\left(\mathrm{cm} \mathrm{H} \mathrm{H}_{2} \mathrm{O}\right) \dagger$ & $6(6-7)$ & $6(6-7)$
\end{tabular}

${ }^{\star}$ Mean (SD).

$\dagger$ Median (interquartile range).

$\ddagger$ Survanta (Ross Laboratories, Columbus, Ohio, USA).

Table 2 Primary and secondary outcomes of the use of binasal and single nasal prongs for delivery of continuous positive airway pressure

\begin{tabular}{|c|c|c|c|}
\hline & Binasal $(n=41)$ & Single $(n=46)$ & p Value \\
\hline \multicolumn{4}{|l|}{ Failure $^{\star}$} \\
\hline All & $10(24)$ & $26(57)$ & 0.005 \\
\hline$<800 \mathrm{~g}$ & $4 / 17(24)$ & $14 / 16(88)$ & $<0.001$ \\
\hline $800-999 \mathrm{~g}$ & $6 / 24(25)$ & $12 / 30(40)$ & 0.384 \\
\hline \multicolumn{4}{|l|}{ Endotracheal reintubation ${ }^{\star}$} \\
\hline All & $9(22)$ & $19(41)$ & 0.089 \\
\hline$<800 \mathrm{~g}$ & $3 / 17(18)$ & $10 / 16(63)$ & 0.023 \\
\hline $800-999 \mathrm{~g}$ & $6 / 24(25)$ & $9 / 30(30)$ & 0.919 \\
\hline Death & $3(7)$ & $2(4)$ & 0.895 \\
\hline Death or in $\mathrm{O}_{2}$ at 28 days & $25(61)$ & $33(72)$ & 0.404 \\
\hline Death or in $\mathrm{O}_{2}$ at 36 weeks & $20(49)$ & $28(61)$ & 0.360 \\
\hline Grade 3 or 4 IVH & $3(7)$ & $2(4)$ & 0.895 \\
\hline PVL & $0 / 40(0)$ & $3 / 46(7)$ & 0.291 \\
\hline Any ROP & $25 / 38(66)$ & $26 / 45(58)$ & 0.602 \\
\hline Grade 3,4 , or 5 ROP & $8 / 38(21)$ & $13 / 45(29)$ & 0.572 \\
\hline Sepsis & $20(49)$ & $22(48)$ & 1.000 \\
\hline Suspected sepsis & $19(46)$ & $23(50)$ & 0.900 \\
\hline Feeding intolerance ${ }^{\star}$ & $19(46)$ & $17(37)$ & 0.503 \\
\hline Weight gain extubation to discharge (g/day) $\dagger$ & $21(7)$ & $20(6)$ & 0.442 \\
\hline Days on IPPV/HFO/NCPAP $\ddagger$ & $35(18-65)$ & $45(29-69)$ & 0.276 \\
\hline Days on IPPV/HFO $\ddagger$ & $8(2-23)$ & $13(3-27)$ & 0.412 \\
\hline Days at level 3 centre $\neq$ & $76(47-107)$ & $73(49-102)$ & 0.902 \\
\hline
\end{tabular}

Unless otherwise indicated, data are expressed as $\mathrm{n}$ (percentage). $\mathrm{p}$ Values in bold are significant. *During week after extubation.

tMean (SD).

$\ddagger$ Median (interquartile range).

IVH, Intraventricular haemorrhage; PVL, periventricular leucomalacia; ROP, retinopathy of prematurity; IPPV, intermittent positive pressure ventilation; HFO, high frequency oscillation; NCPAP, nasal continuous positive airway pressure.

There were no significant differences in the baseline variables known to influence neonatal outcome (table 1). Babies reached minimal levels of ventilatory support and were extubated at a median of 3 days of age (table 1).

An external data monitoring committee performed the first planned interim analysis after 43 patients. This showed no significant difference between the two groups using the O'Brien Fleming criteria. ${ }^{4}$ However, the committee recommended that enrolment be discontinued after the second interim analysis when the $p$ value was 0.005 for the primary outcome measure.

Infants randomised to binasal CPAP were less likely to reach the failure criteria than those allocated to single prong (table 2). Not all infants reaching the preset failure criteria were reintubated, and, although fewer infants in the binasal group were reintubated ( $22 \%$ v $41 \%)$, this result did not reach significance $(p=0.089)$. Within prespecified birth weight strata, failure and reintubation rates were less in the binasal group. These results were statistically significant only for infants $<800 \mathrm{~g}$.

There was no significant difference in the incidence of death or chronic lung disease at either 28 days or 36 weeks of corrected age.
Likewise there were no differences in the rates of cerebroventricular haemorrhage, periventricular leucomalacia, retinopathy of prematurity, sepsis, or feeding intolerance. Weight gain was similar in both groups. There were no significant differences in total duration of assisted ventilation, duration of endotracheal intubation, or stay in the level 3 centre.

\section{Discussion}

Although a simpler and less invasive form of assisted ventilation of the newborn, the introduction of nasal CPAP followed that of intermittent positive pressure ventilation. Gregory et $a \bar{P}$ first described two methods of delivery of CPAP in 1971 for the treatment of RDS: through an endotracheal tube and through a pressure chamber around the infant's head. Subsequently, delivery through a face mask $^{6}$ and nasal tubes ending above the vocal cords have more commonly been used to deliver CPAP. ${ }^{7}$ Several forms of these tubes exist, some long enough to end just above the epiglottis (nasopharyngeal) and some only $1-2 \mathrm{~cm}$ inside the nose. Short prongs may be single or binasal. The Infant Flow Driver (IFD; Electro Medical Equipment, Brighton, Sussex, UK) is a binasal system commonly used in the United Kingdom. In contrast with other binasal systems, it uses a "fluidic flip" mechanism, which is claimed to lead to a more stable level of CPAP being delivered throughout the respiratory cycle. A two hour crossover study showed no real difference between the oxygen requirements or cardiorespiratory variables of infants managed with the IFD and those of infants managed with a single prong CPAP tube. ${ }^{8}$ These findings were supported by a nonrandomised cohort study, which concluded that the IFD offered no short term advantage over single nasal prong CPAP. ${ }^{9}$ We could find no randomised trials comparing different CPAP delivery devices that reported appropriate long term outcomes. Single short prong CPAP had been used for more than 10 years at our centre. Our choice of an alternative CPAP device was influenced by cohort studies reporting the successful use of Hudson prongs in other units. ${ }^{10}$

This study shows that binasal (Hudson) prongs are more effective in preventing extubation failure of ELBW infants than single short nasal prongs. Specific failure criteria (apnoea, respiratory acidosis, and increased oxygen requirements) were chosen as the primary outcome, rather than the other clinically important outcome of endotracheal reintubation, to reduce bias. A randomised trial of these interventions could not be blinded. The decision to reintubate an infant is subjected to potential bias on the part of the clinician making the decision; for example, a clinician with a strong preference for one CPAP device may persist with that device rather than reintubate but elect for earlier intubation if the infant was receiving the other form of CPAP. The outcomes documented on an infant's observation sheet allowed a more objective assessment of an infant's performance to be obtained. 
In a previous trial comparing single prong CPAP with headbox oxygen for care after extubation, we found a high rate of failure of extubation $(91 \%)$ in infants of birth weight below $800 \mathrm{~g}$ randomised to CPAP, and no advantage of CPAP over a headbox in this subgroup. ${ }^{11}$ Stratification by birth weight was performed in the present study to ensure balance between the groups for this important prognostic variable. Subanalysis on the basis of birth weight showed an advantage for binasal prongs in both strata but a statistically significant difference in the lighter infants only.

For the study group as a whole and for the stratum of heavier infants, a trend favouring binasal prongs was seen for the outcome of reintubation. This approached but did not reach significance $(p=0.089)$. A significant result favouring binasal prongs was seen in the stratum of lighter infants. There appears to be a clear message in the high failure rates seen in the smallest babies randomised to single prong CPAP in both studies. It is likely that these infants with their lower respiratory reserves are the most sensitive detectors of a treatment effect. The reasons for the superiority of the binasal prongs are unclear. It is possible that sealing both nostrils results in better transmission of pressure to the upper airways and the lungs.

There were no indications from the other secondary outcomes that the benefits of binasal prongs were accompanied by unwanted side effects.

This study is only one of a number of comparisons of CPAP delivery devices possible. A variety of single and double prong and nasopharyngeal devices have been used. Further randomised trials are required to determine which is superior. It is likely that improvements in the design of CPAP devices will occur that will refine CPAP administration and make it more comfortable for infants. Binasal Hudson prongs are an appropriate benchmark against which newly developed CPAP interfaces may be compared.

CONCLUSION

For ELBW infants ventilated using an endotracheal tube, nasal CPAP delivered through binasal (Hudson) prongs is more effective in preventing failure of extubation than that delivered through a single nasal prong.

The authors are grateful for the assistance of the external monitoring committee, Professor Judith Lumley and Professor David Henderson-Smart. The Royal Women's Hospital Research Foundation funded the study.

1 Davis PG, Henderson-Smart DJ. Nasal continuous positive airways pressure immediately after extubation for preventing morbidity in preterm infants. Cochrane Database Syst Rev 2000;2:CD000143.

2 Papile LA, Burstein J, Burstein R, et al. Incidence and evolution of subependymal and intraventricular hemorrhage: a study of infants with birth weights less than 1,500 gm. $\mathcal{f}$ Pediatr 1978;92:529-34.

3 Anonymous. An international classification of retinopathy of prematurity. The Committee for the Classification of Retinopathy of Prematurity. Arch Ophthalmol 1984;102:1130-4.

4 O'Brien PC, Fleming TR. A multiple testing procedure for clinical trials. Biometrics 1979;35:549-56.

5 Gregory GA, Kitterman JA, Phibbs RH, et al. Treatment of the idiopathic respiratory-distress syndrome with continuous positive airway pressure. $N$ Engl $\mathcal{f} \mathrm{Med}$ $1971 ; 284: 1333-40$

6 Allen LP, Reynolds ER, Rivers RP, et al. Controlled trial of continuous positive airway pressure given by face mask for
hyaline membrane disease. Arch Dis Child 1977;52:373-8.

7 Morley C. Continuous distending pressure. Arch Dis Child Fetal Neonatal Ed 1999;81:F152-6.

8 Ahluwalia JS, White DK, Morley CJ. Infant Flow Driver or single prong nasal continuous positive airway pressure: short-term physiological effects. Acta Paediatr 1998;87:325-7.

9 Kavvadia V, Greenough A, Dimitriou G. Effect on lung function of continuous positive airway pressure administered either by infant flow driver or a single nasal prong. Eur F Pediatr 2000;159:289-92.

10 Avery ME, Tooley WH, Keller JB, et al. Is chronic lung disease in low birthweight infants preventable? A survey of 8 centres. Pediatrics 1987;79:26-30.

11 Davis P, Jankov R, Doyle L, et al. Randomised, controlled trial of nasal continuous positive airway pressure in the extubation of infants weighing 600 to 1250 g. Arch Dis Child Fetal Neonatal Ed 1998;79:F54-7.

\title{
7th European Forum on Quality Improvement in Health Care
}

\author{
21-23 March 2002 \\ Edinburgh, Scotland
}

We are delighted to announce this forthcoming conference in Edinburgh. Authors are invited to submit papers (call for papers closes on Friday 5 October 2001) and delegate enquiries are welcome.

The themes of the Forum are:

- Leadership, culture change, and change management

- Achieving radical improvement by redesigning care

- Health policy for lasting improvement in health care systems

- Patient safety

- Measurement for improvement, learning, and accountability

- Partnership with patients

- Professional quality: the foundation for improvement

- Continuous improvement in education and training

- People and improvement.

Presented to you by the BMJ Publishing Group (London, UK) and Institute for Healthcare Improvement (Boston, USA). For more information contact: quality@bma.org.uk or look at the website www.quality.bmjpg.com. Tel: +44 (0)20 7383 6409; fax: +44 (0)20 73736869. 